\title{
SISTEMA DE GESTIÓN DEL CONOCIMIENTO BASADO EN ESTÁNDARES DE ALTA CALIDAD PARA UN PROGRAMA EDUCACIÓN SUPERIOR*
}

\author{
Knowledge management system based on high quality \\ standards for a higher education program
}

CRISTIAN FELIPE CASTAÑO-DEVIA ${ }^{1}$, GERALDIN QUINTERO-RUIZ², EDGAR DUARTE-FORERO ${ }^{3}$

Recibido:03 de junio de 2021. Aceptado:13 de julio de 2021

DOI: http://dx.doi.org/10.21017/rimci.2021.v8.n16.a102

\begin{abstract}
Resumen
La gestión del conocimiento es una herramienta útil para la competitividad de muchas organizaciones. El sector de la educación superior está particularmente orientado a la adhesión a este tipo de esquemas, ya que su naturaleza implica un constante aprendizaje institucional basado no solamente en su propia experiencia sino en el intercambio de saberes en el ámbito académico. En Colombia, las instituciones de educación superior ven en los estándares de alta calidad del Consejo Nacional de Acreditación una oportunidad de mejora que requiere una infraestructura orientada a la gestión estratégica del conocimiento. Este artículo presenta la experiencia de implementación de un prototipo de sistema de gestión del conocimiento en un programa de pregrado de educación superior. Se describen las fases del proceso y su relación con la plataforma tecnológica que dio soporte al desarrollo. Las conclusiones destacan las ventajas del ejercicio haciendo énfasis en el aprendizaje institucional, la importancia de la plataforma tecnológica y finalmente se plantean reflexiones sobre la gestión del conocimiento como eje fundamental de los procesos de acreditación.

Palabras clave. Gestión del conocimiento; Acreditación; Sistemas de información; Educación superior.
\end{abstract}

\begin{abstract}
Knowledge management is a useful tool for the competitiveness of many organizations. The higher education sector is particularly oriented towards adherence to this type of schemes, since its nature implies constant institutional learning based not only on its own experience but also on the exchange of knowledge in the academic environment. In Colombia, higher education institutions perceive an opportunity for improvement in the high quality standards of the National Accreditation Council, which requires an infrastructure oriented to the strategic knowledge management. This article presents the experience of implementing a prototype of a knowledge management system in a higher education undergraduate program. It describes the phases of the process and its relationship with the technological platform that supported the development. The conclusions highlight the advantages of the exercise, emphasizing institutional learning, the importance of the technological platform and, finally, reflections on knowledge management as a fundamental axis of accreditation processes.
\end{abstract}

Keywords. Knowledge management; Accreditation; Information Systems; Higher Education.

\section{INTRODUCCIÓN}

L A GESTIÓN del conocimiento (GC) es el proceso organizacional conducente para capturar, alma- cenar, analizar y difundir información concerniente a los objetivos estratégicos de un grupo de interés. "Es la disciplina encargada de diseñar e implementar modelos de gestión que permiten

* Artículo desarrollado del proyecto $<<$ Desarrollo del sistema de gestión del conocimiento del programa de ingeniería industrial basado en los lineamientos para la acreditación de programas de pregrado del consejo nacional de acreditación $>>$ ejecutado entre junio de 2020 y mayo 2021 desde el programa de Ingeniería industrial de la Universidad Libre, sede Bogotá.

[1] Ingeniería Industrial, Estudiante de la Facultad de Ingeniería, Universidad Libre Bogotá - Colombia ID: https://orcid.org/0000-0002-7088-2059

[2] Ingeniería Industrial, Estudiante de la Facultad de Ingeniería, Universidad Libre Bogotá - Colombia ID: https://orcid.org/0000-0001-8203-0796

[3] MSc., profesor de la Facultad de Ingeniería, Universidad Libre Bogotá - Colombia ID: https://orcid.org/0000-0001-8072-6976 
identificar, capturar y compartir el conocimiento entre los miembros de la organización, impulsando la creación de valor y generación de ventajas competitivas" [1]. En el marco de la sociedad del conocimiento, este proceso adquiere un rol fundamental para toda organización ya que condiciona la posibilidad de alcanzar ventajas competitivas a partir de la forma como se procese la información asociada al entorno empresarial.

Es necesario reconocer la diferencia entre los conceptos de "conocimiento" e "información". Chiavenato indica que "la información es un mensaje con significado en un determinado contexto, disponible para uso inmediato y que proporciona orientación a las acciones por el hecho de reducir el margen de incertidumbre con respecto a nuestras decisiones" [2]. Por otro lado, la definición de conocimiento es planteada por Canals como sigue: “... es un recurso que no tan sólo nos permite interpretar nuestro entorno, sino que nos da la posibilidad de actuar. Es un recurso que se halla en las personas y en los objetos -físicos o no- que estas personas utilizan, pero también en las organizaciones a las que pertenecen, en los procesos y en los contextos de dichas organizaciones" [3].

La GC no es ajena a las instituciones de educación superior (IES). Por su naturaleza, estas entidades tienen retos estratégicos en lograr una adecuada gestión de sus recursos que asegure un eficiente aprendizaje organizacional. Muchas IES en Colombia evidencian dificultades como pérdida de memoria institucional, disponibilidad y accesibilidad a la información, brechas en la transmisión de información y subvaloración del capital intelectual, entre otros.

Uno de los escenarios que pone a prueba los sistemas de GC para las IES tiene que ver con los procesos de acreditación en alta calidad estipulados por el Consejo Nacional de Acreditación (CNA). El estudio de González-Campo y otros tuvo en cuenta la opinión de más de 200 directivos de IES en Colombia [4]. Sus conclusiones establecen que existe una correlación entre la acreditación institucional de alta calidad y la gestión del conocimiento de las IES. Adicionalmente, el estudio señala que la fase de aplicación de la GC es la que presenta mayores restos y dificultades.

Los sistemas de GC son herramientas de apoyo en la toma de decisiones en organizaciones basa- das en el conocimiento tales como los programas académicos de educación superior. Un caso referenciado en la literatura es el de la Universidad de Southern Queensland [5] en donde la implementación del Sistema de Gestión del conocimiento (SGC) basado en tecnologías de la información y la comunicación ha permitido una mayor gobernabilidad de las directivas y por ende un incremento en la cantidad de proyectos sobre herramientas para la trasferencia del conocimiento.

La estrategia de divulgación del conocimiento es parte fundamental en el desarrollo de las IES [6]. Su implementación requiere del uso de nuevas tecnologías que faciliten el almacenamiento, transformación y divulgación del conocimiento. Por un lado, la habilitación de mecanismos de captura y consulta facilitan una toma de decisiones informada y, en consecuencia, más consciente de las necesidades de la organización. Además, si se complementa con la habilitación de múltiples canales para la difusión del conocimiento, se favorece la transparencia en los procesos de la organización.

Dentro de las tecnologías disponibles para apoyar las estrategias de gestión del conocimiento, la disponibilidad de servicios de colaboración en la nube permite que las organizaciones basadas en el conocimiento promuevan los flujos de información al tiempo que agregan valor a sus procesos. Estos sistemas facilitan el acceso a usuarios de multinivel (generadores, analistas, consultores) bajo criterios de seguridad. La plataforma Microsoft SharePoint, utilizada por Universidad de Southern Queensland [5], ha demostrado ser útil como herramienta para la implementación de sistemas de gestión del conocimiento.

Los antecedentes mencionados conllevan al planteamiento de la siguiente pregunta: ¿Cuáles son las estrategias, mecanismos e infraestructura necesaria para que una IES pueda articular su SGC con el mejoramiento continuo de sus procesos académicos? Para darle respuesta, el artículo se propone como objetivo la propuesta metodológica para la implementación de un SGC en un programa de educación superior en el nivel de pregrado bajo los lineamientos de alta calidad del Consejo Nacional de Acreditación (CNA).

El documento está organizado de la siguiente manera. En la sección número dos se presenta una

Rev. Ingeniería, Matemáticas y Ciencias de la Información Vol. 8 / Núm. 16 / julio - diciembre de 2021; 55-64 
revisión de literatura acerca del uso de la GC soportada por herramientas tecnológicas en IES. Posteriormente, en la tercera sección se describe la propuesta metodológica a través de cinco fases con las que se abordó la problemática. Seguidamente se exponen los resultados alcanzados, y se exponen las conclusiones y recomendaciones extraídas del proceso adelantado.

\section{REVISION DE LITERATURA}

En esta sección se realiza un barrido por algunos referentes importantes en materia del desarrollo e implementación de sistemas de gestión del conocimiento en IES. La revisión de literatura permitió validar la importancia de estos tipos de sistemas de gestión y su carácter estratégico en el campo de acción referido.

En 2018, Vaquiro [7] identificó como se desarrollan las prácticas de gestión del conocimiento en 100 instituciones de educación en el departamento del Huila, con el apoyo de 210 personas entre directivos, docentes de básica y media. Dentro de los resultados se encontró que las instituciones educativas estudiadas transformaron sus prácticas educativas en procesos de gestión de conocimiento, sin una preferencia específica sobre los mecanismos para hacerlo. No obstante, se encontró una predominancia en las subcategorías de adquisición, diseminación de la información, e interpretación compartida. Adicionalmente, el estudio concluye indicando la importancia de mejorar en los procesos de almacenamiento, transferencia, uso y aplicación del conocimiento.

Para proponer un SGC es necesario hacer un análisis situacional como lo indica Uribe [8] en su estudio realizado en IES de Medellín. Además de presentar una caracterización que permitió identificar los impactos, niveles de implementación y vacíos de los sistemas examinados. El autor argumenta que las IES se ven afectadas en el proceso de la trasferencia de la información por los bajos niveles de madurez de los SGC existentes.

Rowley señala que si bien las IES cuentan con instalaciones, sistemas y proyectos que les ayudan en el manejo de su información (bibliotecas, intranets, plataformas de aprendizaje, repositorios digitales), persisten desafíos en la creación de un entorno de conocimiento [9]. Al lograr ese entorno las IES pueden hacer que sus procesos de gestión del conocimiento alcancen cambios importantes en la cultura, valores y estructuras organizativas.

Según González Campo y otros [4] cuentan como el CNA en Colombia es el encargado de liderar los procesos de acreditación de calidad siendo la institución más antigua de Suramérica y convirtiéndose en un referente internacional por su solidez e integralidad. Esta evaluación se realiza con el fin de evidenciar altos niveles de calidad e implementar modelos de gestión del conocimiento para facilitar la acreditación, la evaluación cuenta con 40 indicadores que miden cada sub-proceso de acuerdo al proceso general de enseñanza- aprendizaje. Los resultados de este estudio indican que la acreditación de alta calidad impulsa la gestión del conocimiento y los mejores resultados se evidencian de instituciones que cuentan con este sistema.

Con respecto a la propuesta de metodologías para la implementación de un SGC, Acevedo Correa y otros propone cuatro fases: diagnóstico, diseño, implementación y validación de estrategias [10]. El estudio permite entender la utilidad de realizar un diagnóstico previo con el fin de contextualizar el panorama de intervención. Adicionalmente, la implementación del modelo ayuda a orientar al fortalecimiento de la cultura alrededor de estrategias de preservación de conocimiento lo cual se relaciona con la creación, codificación, apropiación y la reutilización de los conocimientos ya existentes. Este ejercicio conlleva a la consolidación de experiencias que fomentan la apropiación y transferencia de conocimiento.

Las herramientas tecnológicas tienen un rol protagónico en la GC. Lindvall y Rus [11] presentan una introducción acerca a la GC en la ingeniería del software. A partir de la identificación de necesidades del conocimiento, elementos claves y origen del conocimiento, analizar la relevancia que tiene la captura, organización, recuperación y acceso a la información evitando así la pérdida de información.

La adopción de herramientas tecnológicas tiene el potencial para que la gestión del conocimiento sea eficiente y eficaz al momento de ejecutar. Arpaci [12] indaga acerca de los antecedentes y 
consecuencias que conlleva implementar los sistemas de gestión del conocimiento que manejen su información en la nube. Por medio de una encuesta realizada a 221 estudiantes de pregrado examinó la relación entre las expectativas de la práctica de gestión del conocimiento y la utilidad generada de los servicios de computación en la nube. Los hallazgos comprobaron que el uso intensivo de herramientas tecnológicas en las IES favorece la utilidad de la GC.

Finalmente, es relevante establecer la proyección que tienen los sistemas de GC a la luz de la influencia de la pandemia de Covid 19 en los entornos educativos. El estudio de Barón y Caicedo [13] resalta la importancia de una transformación digital como soporte a la ruptura de desigualdades evidenciadas por la pandemia. Sin ayuda de las herramientas tecnológicas, el aprendizaje no se llevaría de forma continua, convirtiéndose en un factor clave en el aporte de calidad y cobertura en la educación.

\section{Materiales Y MÉTODOS}

Para el desarrollo del presente estudio se diseñó un proceso mixto con componentes exploratorios y descriptivos. Este proceso fue aplicado en un objeto de estudio identificado como un programa de pregrado de una universidad acreditada que funciona en la ciudad de Bogotá. Como se mencionó en el objetivo, el proceso de investigación se orientó a la implementación de un SGC enfatizado en los factores de acreditación del CNA para pro-

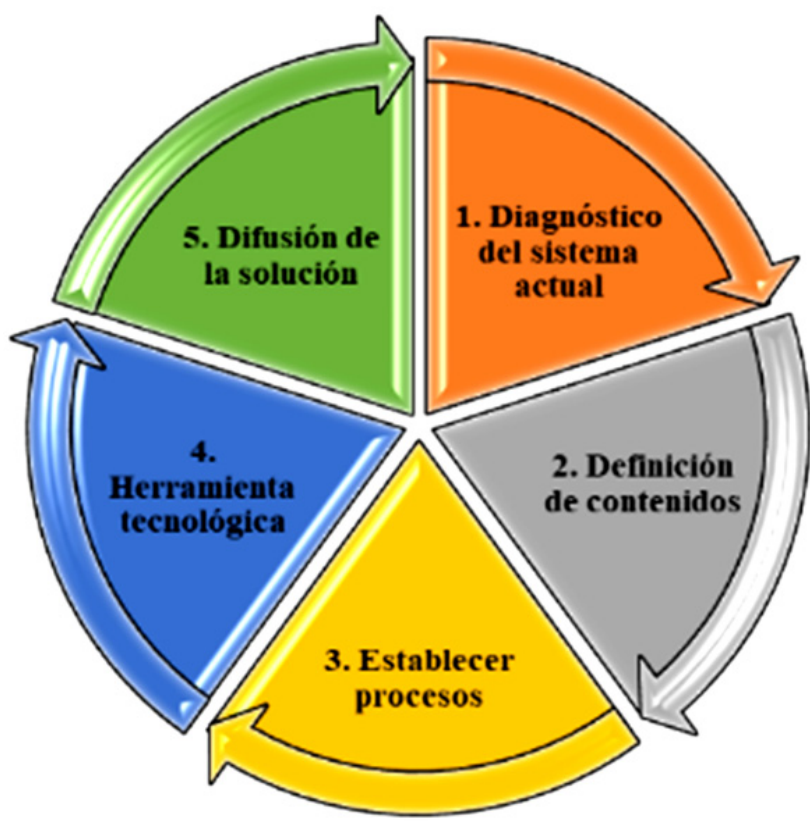

Fig. 1. Fases aplicadas durante el desarrollo del proyecto.

gramas de pregrado. La implementación desarrollada tuvo como alcance los factores "Estudiantes" y "Docentes". El desarrollo del proyecto se llevó a cabo en cinco fases (Fig. 1).

\subsection{Diagnóstico del sgc para programa de pregrado}

Con el fin de diagnosticar el nivel de apropiación de la comunidad académica acerca del concepto de GC, se realizó una encuesta en la que participaron docentes, estudiantes y personal administrativo de la institución (Ver Fig. 2) .

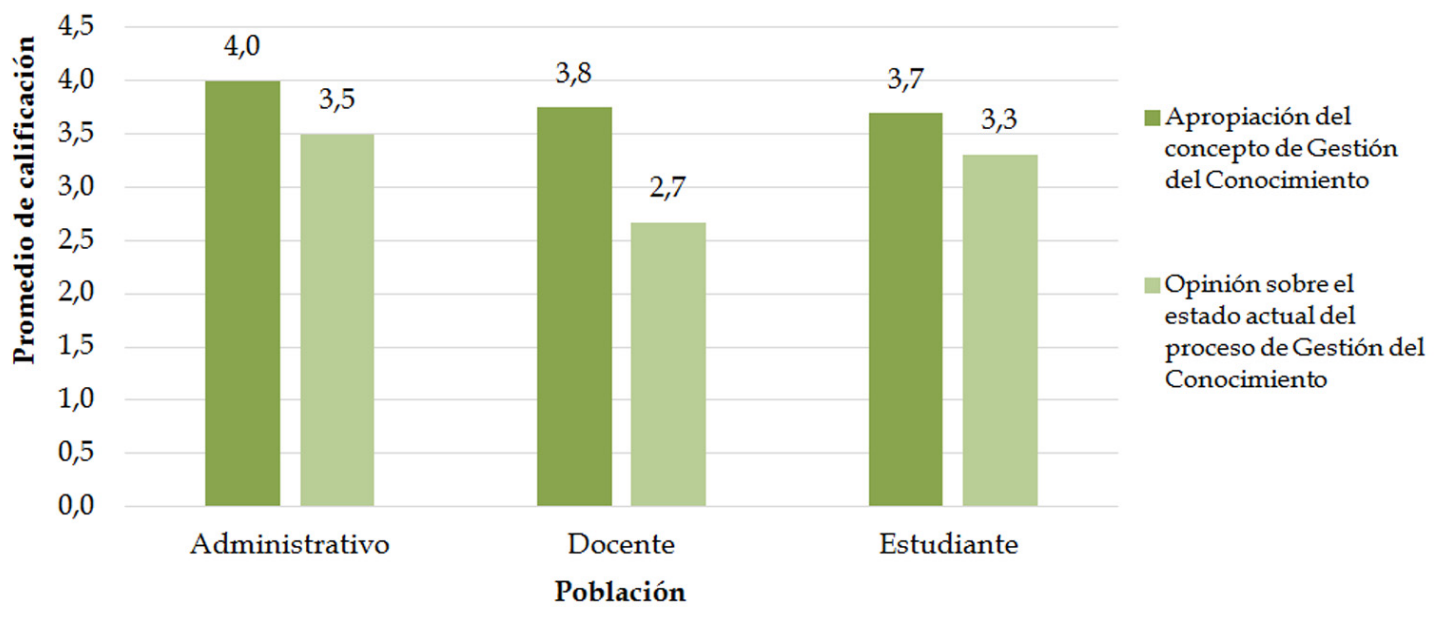

Fig. 2. Calificación el proceso y dominio del concepto de gestión del conocimiento. 


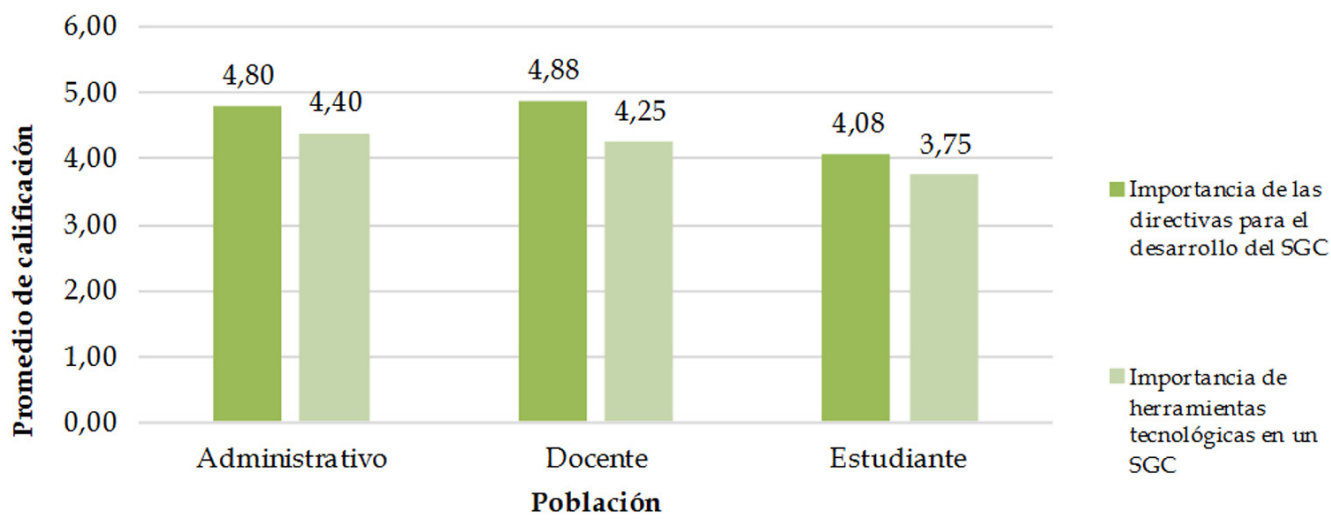

Fig. 3. Apreciación sobre la implementación del sistema de Gestión del Conocimiento.

Los resultados de la encuesta indican que la población domina el concepto de gestión del conocimiento, así como sus beneficios en la eventual implementación en un programa de educación superior. También manifestaron preocupación por el actual manejo de la información pues se evidencian discontinuidades y fragmentación en los procesos de transmisión.

En la Fig. 3 , se evidencia la apreciación de la comunidad académica sobre la importancia del apoyo de directivas en la construcción del SGC. También se valoró el rol de las herramientas tecnológicas en la construcción de soluciones que apoyen al SGC. Este ejercicio de diagnóstico se complementó con la caracterización de los sistemas de información existentes en la institución, los mecanismos de articulación y la utilidad que éstos presentan para la toma de decisiones.

\subsection{Definición de contenidos relevantes}

El SGC debe funcionar alrededor de unos objetivos propuestos, el tipo de información existente y las personas que participan en el proceso de generación de conocimiento. Para ello se definió que la información a administrar será aquella relacionada con los factores de "Docentes" y "Estudiantes" definidos por estándar de alta calidad del Consejo Nacional de Acreditación [14]. El estándar describe cada factor estableciendo los documentos e indicadores cuantitativos, que permiten evidenciar las acciones de mejora correspondientes.

Se establecieron las fuentes de información que nutren cada factor. La clasificación de los conteni- dos requirió asignar identificadores que permitieran comprender su naturaleza y relación con el estándar CNA en términos de factores, características y aspectos a evaluar. En este sistema se proponen cinco tipos de contenidos: documentos, indicadores estadísticos, resultados de encuestas, opiniones de paneles de expertos e hitos destacados.

El tipo de contenido documental tiene que ver con normas, reglamentos, actas o archivos de información escrita y legible. Los indicadores estadísticos se refieren a variables cuantitativas asociadas con relaciones de profesores por estudiante, actividades de semilleros o grupos de investigación, entre otros. El tercer tipo de contenido, respuesta de encuestas, considera la sistematización de las apreciaciones de autoevaluaciones que periódicamente realiza el programa académico. En muchos casos, el programa acude a paneles de expertos como fuentes de información para la toma de decisiones, cuyos resultados son configurados también en el SGC. Finalmente, el SGC también contempla la posibilidad de registrar hitos de eventos, actividades, reconocimientos, y otras acciones relevantes, como un tipo de contenido.

Para acceder fácilmente a la información se creó una codificación para cada tipo de contenido que contempla el factor, característica y aspecto a evaluar asociados. Por ejemplo, el factor "Estudiantes" es codificado con número "02" según los lineamientos del CNA. Lo propio se desarrolla con las características y aspectos a evaluar. A manera de ejemplo, todos los contenidos de información etiquetados en el SGC con el código "F02C07A037" corresponden al Factor "02 Estudiantes", a la Ca- 
racterística “07 Estímulos y apoyos a estudiantes”, y al Aspecto a evaluar "037 Impacto de la aplicación de las políticas y estrategias sobre estímulos académicos y apoyos socioeconómicos para los estudiantes, que atienden a la diversidad, pluralismo e inclusión". La Fig. 4 representa un ejemplo de esta estructura.
La definición de contenidos relevantes se concreta en un cuadro maestro de contenidos. El cuadro maestro define qué tipo de contenidos son manejados para cada factor, característica y aspecto a evaluar (Fig. 5). De manera complementaria, determina los niveles de responsabilidad, repositorios y frecuencias de actualización.

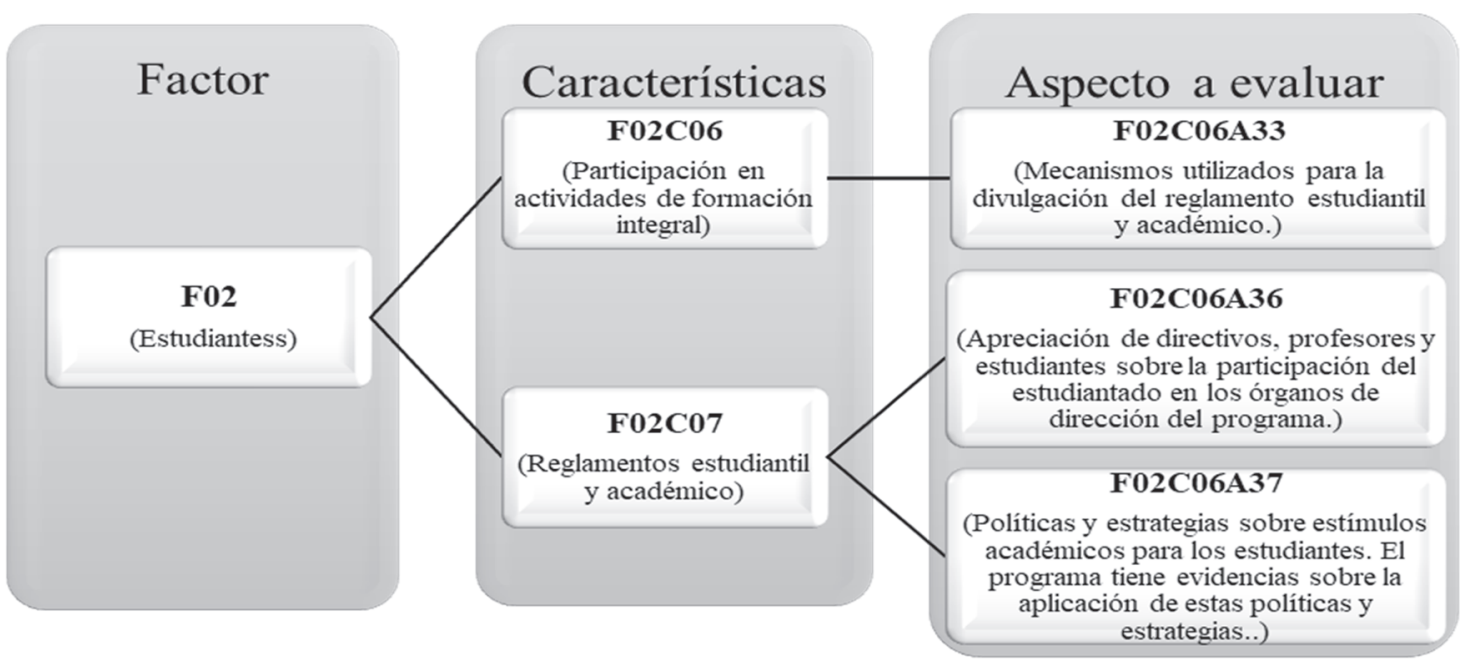

Fig. 4. Codificación Jerárquica.

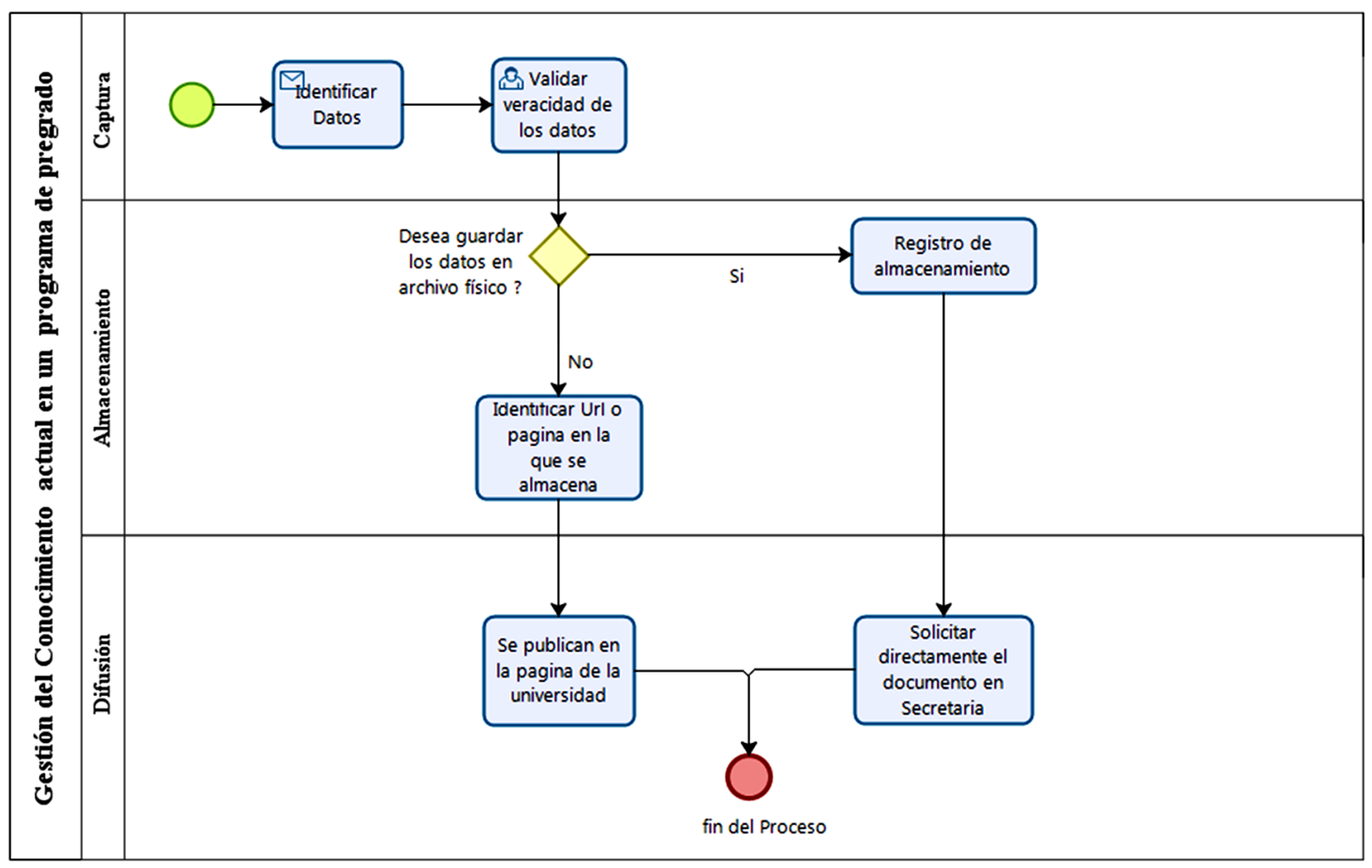

Fig. 5. Proceso de Gestión del conocimiento actual en un programa académico. 


\subsection{Procesos para la implementación del SGC}

Los diagramas Business Process Management (BPM) permiten identificar los flujos de información al interior de una organización. Para identificar estos flujos se construyeron diagramas BPM para todos los procesos que tuvieran que ver con los factores "Docentes" y "Estudiantes". Los diagramas resultantes permitieron reconocer los contenidos específicos necesarios para cumplir con los requisitos del estándar de acreditación del CNA. Además, se identificaron los responsables, fuentes de información, periodicidad de generación, medio de divulgación y repositorio existente.

Con la construcción de diagramas de flujos en formato BPM se identificó el proceso de recolección, almacenamiento y difusión actual de la información. En su construcción se identificaron los ajustes necesarios al proceso y la forma de llevarlos a cabo. La Fig. 5 representa de manera genérica el flujo actual de información en el programa académico. Este análisis se llevó a cabo para cada tipo de contenido identificando vacíos en los subprocesos de captura, almacenamiento, difusión.

Tras construir los diagramas BPM se realizó una asociación de perfiles de manejo de información a los cargos relacionados con los factores "Estudiantes" y "Docentes". Se establecieron responsabilidades en áreas como Secretaría Académica, Dirección del Programa, Secretaría General, Área Financiera, Decanatura, Bienestar Universitario entre otras. A partir de allí se llevó a cabo una caracterización de qué documentos existían, dónde se generaban (área procedente), a quién iban destinados, dónde se almacenaban y con qué frecuencia se almacenaban.

El ejercicio permitió depurar los flujos y definir los puntos necesarios de captura, almacenamiento, análisis y difusión, como se muestra en la Fig. 6. A través de entrevistas y sesiones de trabajo compartido se construyó una estructura enriquecida para el flujo de información que sirvió de base para la construcción del SGC.

\subsection{Implementación de proceso en herramienta tecnológica}

Para la implementación del proceso se tuvieron en cuenta los recursos con los que dispone la orga- nización. El uso de aplicativos basados en computación en la nube resulta adecuado por cuanto facilita el acceso, transparencia y seguridad de la información. En el caso específico estudiado, se construyó un sistema de información utilizando la plataforma SharePoint.

Tratándose de un sistema de información para apoyar el SGC, es de gran importancia definir los mecanismos de captura, almacenamiento y difusión de la información. Se diseñaron dos mecanismos de captura de información. En primer lugar, una aplicación construida con Power Apps, permite obtener datos a través de dispositivos móviles. El segundo mecanismo implica el uso de formularios personalizados en Sharepoint que son distribuidos a los usuarios del SGC bajo una estructura de permisos de ingreso, lectura, edición y aprobación.

Una plantilla define los campos que va a tener cada tipo de contenido, mientras que los registros se refieren a las instancias respectivas. Las listas de plantillas contienen la estructura básica que deben tener los tipos de contenidos. Cada plantilla de contenidos está vinculada a una o más codificaciones de factor, característica y aspecto a evaluar según la estructura del estándar de acreditación del CNA. Las listas de registros de contenidos contienen las instancias de los contenidos (Ver Fig. 7). A manera de ejemplo, la lista de "Plantilla de contenidos" puede contener un tipo de contenido denominado "Syllabus". Los "Syllabus" pueden responder a una o más combinaciones de factor, característica y aspecto a evaluar (Lista "Codificación"). Finalmente, en la lista "Registro de contenidos" se ingresan las instancias para los distintos syllabus con los que cuenta el programa.

Las plantillas fueron clasificadas en cuatro categorías: documentos, indicadores, preguntas y opiniones de panel de expertos.

\subsection{Difusión de la información del sistema}

La GC requiere que la información almacenada se difunda estratégicamente para que se pueda convertir en conocimiento de la organización. Para el análisis de la información se diseñaron estándares de indicadores, documentos e informes que facilitan el proceso de aprendizaje organizacional. La herramienta aplicada para este efecto consistió 


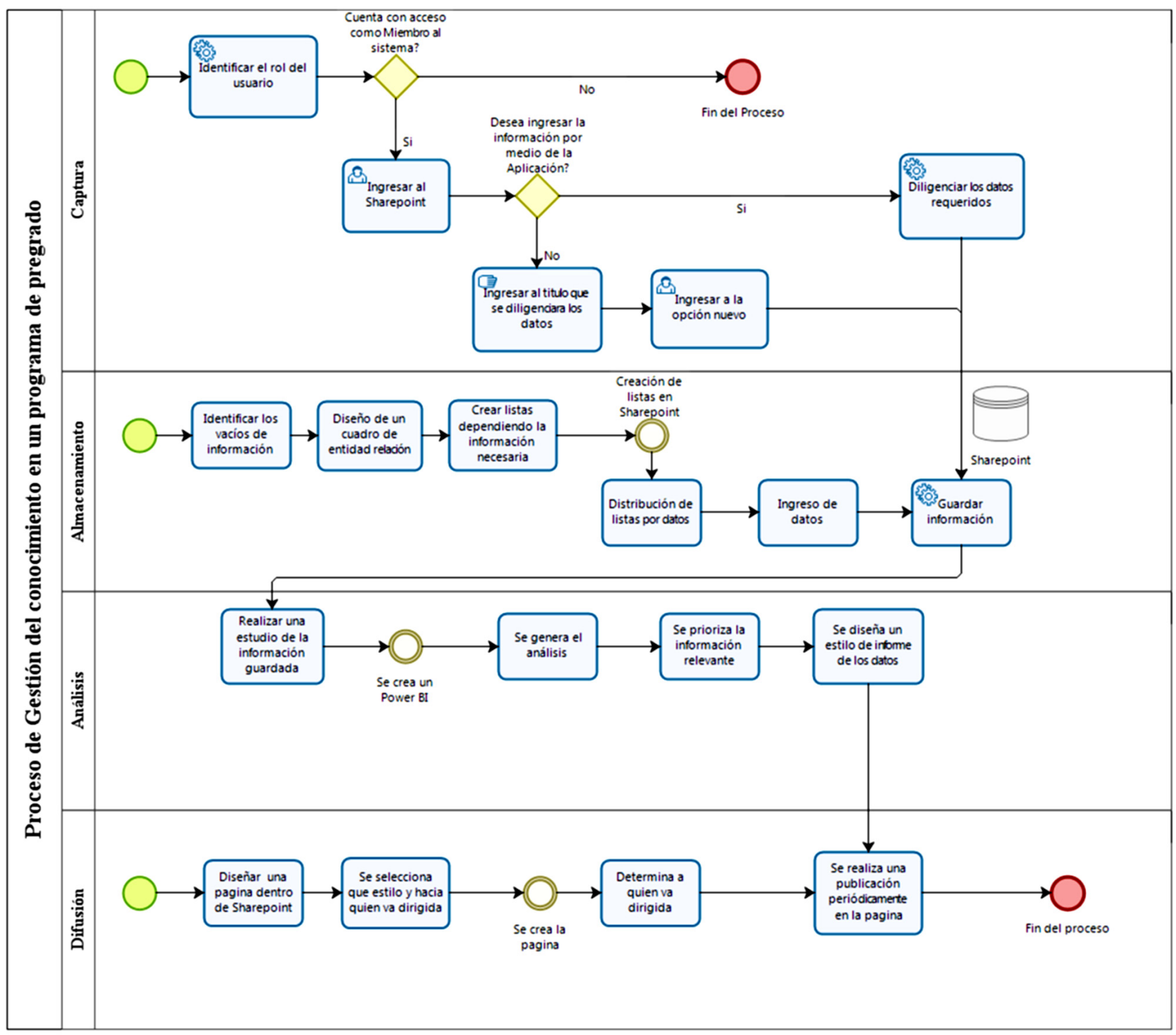

Fig. 6. Proceso de Gestión del Conocimiento propuesto para el programa.

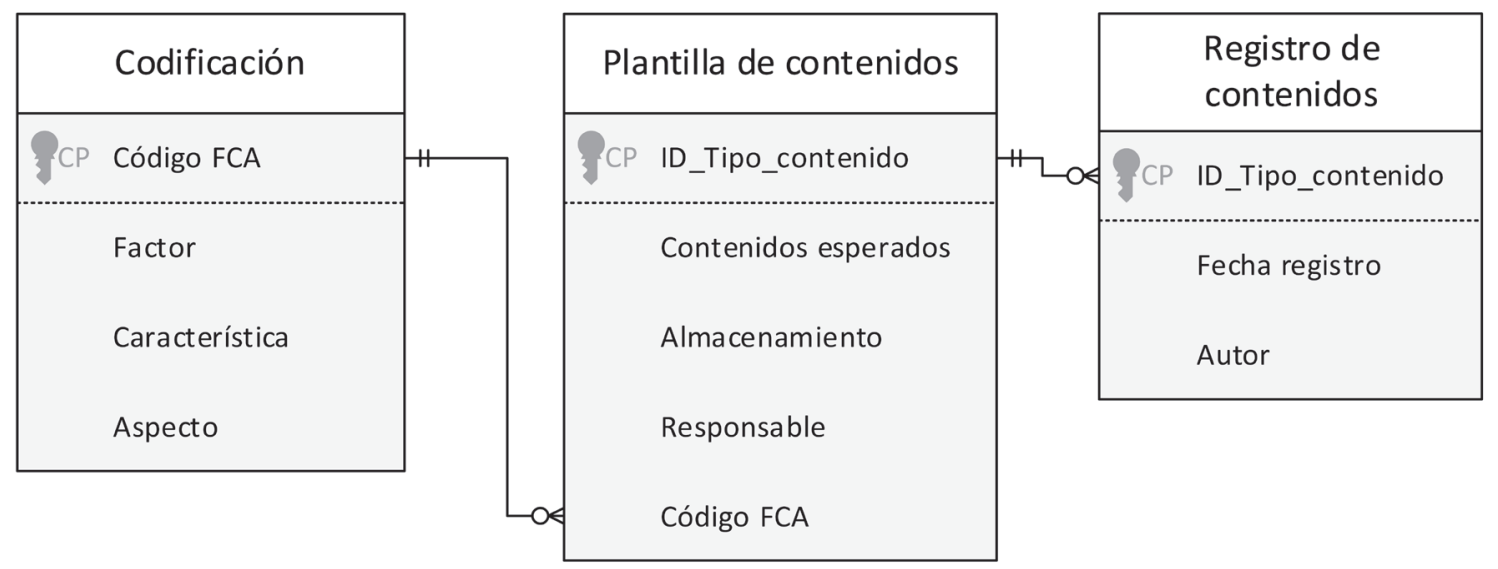

Fig. 7. Esquema básico de la estructura de listas 
en PowerBI, la cual permite tener datos actualizados en tiempo real y difundir contenidos a través de tableros de comando. Adicionalmente, los usuarios autorizados de la organización pueden acceder a las listas construidas en el SGC por medio de la interfaz de SharePoint y configurar los informes requeridos para cada tipo de contenido. Una de las principales ventajas consiste en que estos tipos de contenidos se pueden filtrar por cualquiera de las categorías establecida en el estándar CNA.

Esta etapa de difusión de información está estrechamente atada a la utilidad que tiene el SGC. Por tal razón, no se habilitó un acceso abierto a los contenidos almacenados. En su lugar, se configuraron informes específicos para las actividades concernientes al proceso de acreditación en alta calidad del CNA: ponderación de factores, autoevaluación, reporte de contenidos por factor, acciones por mejorar, diseño de plan de mejora.

\section{Resultados}

La propuesta de constitución de un SGC para el programa académico estuvo compuesta por cinco etapas: diagnóstico, definición de contenidos, definición de procesos, herramienta tecnológica y difusión de la solución. Estos pasos permitieron constituir un SGC que organizado alrededor de los componentes de captura, almacenamiento y difusión de la información. La herramienta utilizada para la configuración del SGC consistió en un sistema de colaboración en la nube operado a través de Sharepoint. Los módulos de captura permiten que los usuarios ingresen información sobre registros asociados al estándar CNA a través de formularios estandarizados.

Durante el proceso de implementación se configuraron los 12 factores, 42 características y 200 aspectos a evaluar en las listas del SGC. El ingreso de la información se llevó a cabo con datos tomados de los últimos dos años de gestión del programa logrando registrar 30 tipos de documento, 15 indicadores y más de 100 registros de distinta índole. Con base en esta información se generaron xxx indicadores del SGC así como otras piezas comunicativas asociadas a la información obtenida.

Es importante mencionar que la validación de la propuesta de SGC se realizó en talleres conjun- tos con estudiantes, docentes y personal administrativo. Estos espacios permitieron identificar opciones de mejora, propuestas para simplificar el proceso y ajustes en los informes a producir. Los talleres desarrollados permitieron dar a conocer las diferentes herramientas y funciones con las que cuenta el SGC. El uso de juegos de roles permitió reconocer la importancia de los distintos roles de acceso a la información (consulta, ingreso y edición) que fueron habilitados para directores, docentes, administrativos y estudiantes. La socialización del SGC involucró además el diseño de videotutoriales sobre los procesos de captura, almacenamiento de datos y difusión de información.

La principal dificultad que se tuvo con esta implementación consistió en el acceso a información solicitada por el estándar CNA. El ejercicio identificó que, si bien existe la información, su acceso es claramente complejo. Con la implementación del SGC se logró facilitar la accesibilidad a la información disponible para los tomadores de decisiones en el programa.

\section{Conclusiones}

Este proyecto es resultado de los planes de mejora construidos por las directivas del programa en el marco de procesos de acreditación previos. En tal ocasión se identificó que una de las oportunidades de mejora consistía en una mejor gestión del conocimiento. Se planteó una metodología para la implementación de un SGC que da soporte al proceso acreditación del programa y facilita a otras áreas el manejo de datos y de información. La metodología está dividida en cinco fases: diagnóstico, definición de contenidos, definición de procesos, herramienta tecnológica y difusión de la solución.

Las fases de diagnóstico, definición de contenidos y definición de procesos estuvieron orientadas a identificar los contenidos que deben ser priorizados, sus características y flujos asociados. Herramientas como entrevistas, levantamiento de diagramas BPM y diseño conceptual de bases de datos permitieron alcanzar el esquema que se concretizó en la fase de herramienta tecnológica. El diseño y mayoría de aspectos de la implementación realizada se construyó sobre la infraestructura de SharePoint, Power Apps y Power BI. 
El sistema de gestión del conocimiento propuesto tuvo impactos preliminares positivos en términos de organización de la información, identificación de perfiles, codificación de la información y mayores contenidos para la toma de decisiones. Además de esto, al encontrarse en un proceso de acreditación del programa se tendrá en cuenta el manejo de la información y el sistema de gestión podrá ser más eficiente.

Futuros trabajos podrán realizar ejercicios para ampliar el alcance del sistema propuesto hacia todos los factores de alta calidad del CNA, así como a la automatización en el flujo de actividades dentro del sistema de información utilizando mecanismos que hacen parte de la plataforma tecnológica. Otro frente de trabajo consiste en la evaluación del impacto en el mediano y largo plazo que pueda tener esta herramienta. Los resultados obtenidos cubren las primeras impresiones de actores vinculados al programa, pero es necesario realizar un escalamiento en el tiempo para reconocer los beneficios y dificultades que brinda en SGC en un plano estratégico.

Finalmente, el equipo de trabajo considera que es pertinente la reflexión acerca de la importancia de la GC en los procesos de acreditación de programas académicos. Siendo organizaciones basadas en el conocimiento que marcan una impronta en las futuras generaciones de profesionales, se hace necesario que se dé una evolución del pensamiento del programa y que esta característica sea gestionada estratégicamente por las instituciones. La GC permite que las organizaciones construyan sobre lo aprendido y amplíen los espectros de sus conocimientos. Los SGC son entonces herramientas que ayudarían a la búsqueda de la alta calidad en organizaciones de esta índole.

\section{REFERENCIAS}

[1] M. Perez Montoro, «Gestión del conocimiento: orígenes y evolución,» Profesional de la información, vol. 25, no. 4 , pp. 526-534, 2016.

[2] I. Chiavenato, Introducción a la Teoría General de la Administración, 7 ed., McGraw-Hill Interamericana, p. 110. 2006.

[3] A. Canals, «La gestión del conocimiento,» in Acto de presentación del libro Gestión del Conocimiento, 2003.
[4] C. González-Campo, G. Murillo-Vargas and M. García-Solarte, «Efecto de la acreditación institucional de alta calidad sobre la gestión del conocimiento,» Formación universitaria, vol. 14, no. 2, pp. 155-164, 2021.

[5] B. Millett, T. Te'o, D. Rhodes, J. Clarke and S. Carswell, «SharePoint portal as a strategic management and planning tool: University of Southern Queensland (USQ) as a case study,» Institutional Research, Innovation and Change in Universities of the 21st Century, 2005.

[6] E. Moresi, «Inteligência organizacional: um referencial integrado.,» Ciência da Informação, vol. 30, no. 2, pp. 35-46, 2001.

[7] M. Vaquiro Motta, Artist, Prácticas de Gestión del conocimiento en las. [Art]. Universidad Católica de Manizales, 2018.

[8] A. F. Uribe Acosta, «La Gestión del conocimiento en Instituciones de Educación Superior (IES) de Medellín,» Institución Universitaria Esumer, pp. 243258, 2013.

[9] J. Rowley, «Is higher education ready for knowledge management?,» International Journal of Educational Management, vol. 14, no. 7, pp. 325-333, 2000.

[10] Y. Acevedo Correa, C. A. Aristizábal Botero, A. Valencia Arias and L. Bran-Piedrahita, «Formulación de modelos de gestión del conocimiento aplicados al contexto de instituciones de educación superior.,» Información tecnológica, vol. 31, no. 1, pp. 103-112, 2020.

[11] M. Lindvall and L. Rus , «Knowledge Management for Software Organizations,» Managing Software Engineering Knowledge, 2001.

[12] I. Arpaci, «Antecedents and consequences of cloud computing adoption in education to achieve knowledge management,» Computers in Human Behavior, vol. 70, pp. 382-390, 2017.

[13] C. Y. Barón Hernandéz and E. Caicedo Rojas, «Transformación digital, un desafío en la educación superior,» Inventum, vol. 16, no. 30, pp. 3-11, 2021.

[14] Consejo Nacional de Acreditación, «Lineamientos y aspectos por evaluar para laacreditación en alta calidad de programas académicos,» Bogotá, 2021.

[15] G. Murillo Vargas, C. H. González Campo and M. García Solarte, «Efecto de la acreditación institucional de alta calidad sobre la gestión del conocimiento,» Formación Universitaria, vol. 14, no. 2, pp. 155-164, 2021. 